\title{
The Mouse Gambling Task: Assessing Individual Decision-making Strategies in Mice Elsa Pittaras ${ }^{1, *}$, Arnaud Rabat ${ }^{2,3, \#}$ and Sylvie Granon, \#
}

${ }^{1}$ Biology Department, Stanford University, Stanford, CA 94305-5020, USA; ${ }^{2}$ Unité Fatigue et Vigilance, Département Environnements Opérationnels, Institut de Recherche Biomédicale des armées, 1 place du Général Valérie ANDRE, BP 73, 91223 Bretigny-sur-Orge cedex, France; ${ }^{3}$ Equipe d'accueil 7330 "VIFASOM», Hôtel Dieu AP-HP, Université Paris 5 Descartes, 1 place du Parvis Notre Dame, 75181 Paris cedex 04, France; ${ }^{4}$ Neurobiology of decision making, Paris-Saclay Institute of Neuroscience (Neuro-PSI), CNRS UMR 9197, 91400 Orsay, France

*For correspondence: pittaras.e@gmail.com

\#Contributed equally to this work

[Abstract] Decision-making is a complex cognitive process which consists of choosing one option among several alternatives. In humans, this process is featured in the lowa gambling task (IGT), a decision-making task that mimics real life situations by reproducing uncertain conditions based on probabilistic rewards or penalties (see Background). Several authors wanted to adapt the IGT in rodents with subtle differences in protocols that match various aspects of the human task. Here we propose, for the first time in mice, a protocol that contains the most important characteristics of the IGT: 4 different options, choices based on 4 ambiguous outcomes with immediate and long term rewards, a total of 100 trials, no learning of the contingency before the task, and presence of both a certain reward and a probable penalty. During this task, mice have to choose between options more or less advantageous in the short and long term by developing a decision-making strategy that differs between individuals. Therefore, the strength of this protocol is that it is one of the first to enable the study of decision-making in a complex situation, and demonstrates inter-individual differences regarding decision-making strategies in mice.

Keywords: Decision-making, Mouse, Inter-individual differences, Gambling, Reward, Maze, Strategies

[Background] Decision-making is a cognitive process which consists of choosing one option among several alternatives. It progresses from the exploration of unknown options to the exploitation of preferred ones (Bechara et al., 1994). During this cognitive process, the decision maker evaluates the value of each option regarding his/her own preferences and the probability to get it. This computation brings him/her to select one strategy among others. Such strategies are featured in the lowa gambling task (IGT), a decision-making task that mimics real life situations by reproducing uncertain conditions based on probabilistic rewards or penalties (Bechara et al., 1994). During this task, subjects have to implicitly discover (through somatic markers) which option is advantageous in the long term, with the progressive discovery that options are not available under fixed and predictable contingencies. Several authors adapted the IGT in rodents (de Visser et al., 2011a; van den Bos et al., 2014) to study neurobiological substrates of decision-making (non-exhaustive exemples: Homberg et al., 2008; Pais- 
Vieira et al., 2009; Zeeb et al., 2009; de Visser et al., 2011a, 2011b and 2011c; Young et al., 2011; Zeeb and Winstanley, 2011; Koot et al., 2012; Pittaras et al., 2013; Rivalan et al., 2013; Peña-Oliver et al., 2014; Van Enkhuizen et al., 2014; Fitoussi et al., 2015; Milienne-Petiot et al., 2017), sex differences (G van den Bos et al., 2012; Georgiou et al., 2018), drugs effect on decision-making (Sanchez-Roige et al., 2015; Gueye et al., 2016; de Laat et al., 2018; Ferland et al., 2017 and 2018), and environmental (Van Hasselt et al., 2012; Koot et al., 2013; Loi et al., 2017a and 2017b) or physiological features on decisionmaking (Pais-Vieira et al., 2009; de Visser et al., 2011b and 2011c; Koot et al., 2012). So far, the existence of inter-individual differences in decision-making has been linked to specific behaviors (Rivalan et al., 2009 and 2013; Ferland and Winstanley, 2017) and differential neuronal (Rivalan et al., 2009; Fitoussi et al., 2015) or regional neurochemical activity (Pittaras et al., 2016a and 2016b). The numerous adaptations were either in an operant chamber (e.g., Rivalan et al., 2009; Zeeb et al., 2009) or in a maze (e.g., van den Bos et al., 2006; Pittaras et al., 2013 and 2016a). Several reviews provide exhaustive details about the differences and the protocols' procedures (e.g., de Visser et al., 2011a; van den Bos et al., 2006 and 2014). The protocol that we propose here matches, for the first time in mice, the most important characteristics of the IGT: 4 different options, choices based on 4 ambiguous outcomes with immediate and long term rewards, a total of 100 trials, no learning of the contingencies before the task, and presence of both a certain reward and a probable penalty (Pittaras et al., 2013, 2016a, 2016b and 2018).

Our protocol allows for a precise analysis of individual decision-making strategies in ambiguous situations in mice. Therefore, it can be used to characterize decision-making deficits in a large number of pathological mouse models and assess putative treatments. Additionally, it can be used to test alteration/improvement of decision-making strategies in extreme situations (e.g., lack of sleep, stress). Another very interesting point of this protocol is that it targets inter-individual variability. Indeed, outside of pathological conditions, risky or rigid decision-making could lead to behaviors that have unhealthy consequences over life (drug taking, car accident, unsuitable behavior, etc.). Therefore, by using this protocol, it is possible to understand the neurobiological basis of such behaviors, which provides insight of how to prevent these behaviors.

\section{Materials and Reagents}

1. 8 standard water bottle caps

2. Paper towel

3. Plastic Pasteur pipette

4. Mice (any strains and sex)

5. Dustless Precision Pellets (BioServ, New Jersey, catalog number: F0163), Grain-based, $20 \mathrm{mg}$

6. Quinine (Quinine hydrochloride dihydrate, Sigma, catalog number : Q1125-10G)

7. Distilled water

8. $10 \%$ ethanol 


\section{Equipment}

1. Balance

2. Timer

3. Skinner cages (optional)

Apparatus: Identical operant chambers $(20 \mathrm{~cm} \times 24 \mathrm{~cm} \times 16 \mathrm{~cm}$, Figure 2A) from ImetronicR (Pessac, France, http://www.imetronic.com/devices/polymodal-system/) are used. Each box includes a house light system delivering approximately 20 lux of white diffused light, 5 holes and a food magazine, located on the wall opposite to the holes, that can deliver food pellets (dustless Precision Pellets, Grain-based, $20 \mathrm{mg}$, BioServ, New Jersey). During habituation only the central hole and the magazine, equipped with infrared beams detecting head entries, are available.

4. Maze with four transparent arms (20 cm long $\times 10 \mathrm{~cm}$ wide), an opaque start box $(20 \mathrm{~cm} \times 20$ $\mathrm{cm})$ and a choice area

\section{Procedure}

The experiment consists of two weeks of habituation in the operant chamber followed by one week of the Mouse Gambling Task in the maze (Figure 1).

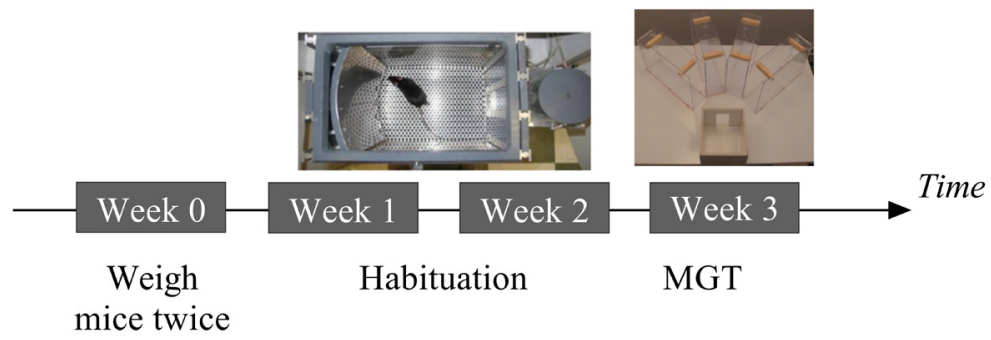

Figure 1. Timeline of the experiments. MGT: Mouse Gambling Task.

A. Animals

1. Depending on your scientific question, this protocol can be done with males or females, different strains and ages or environments (e.g., light cycle), provided that they are mice that can undergo controlled food restriction. In our condition, male C57BI/6J mice that are 3 to 6 months old at the beginning of the experiments (Charles' River, Orleans, France) are housed by three or four, in a temperature-controlled room $\left(21 \pm 2{ }^{\circ} \mathrm{C}\right.$ ), with a 12 light/12 dark cycle (lights on at 08:00 AM). We always perform experiments between 09:00 AM and 06:30 PM. Animals should be treated according to the ethical standards defined by the "Centre National de la Recherche Scientifique" for animal health and care with strict compliance with the EEC recommendations ( ${ }^{\circ} 86 / 609$ ). To perform inter-individual analysis, we advise using approximately 20 to 30 individuals.

2. During the MGT, mice need to be food-restricted (individually maintained at $85 \%$ of the free- 
feeding weight). Water is always provided ad libitum. The week before starting food restriction, weigh the animals twice (two different days, free-feeding weight) and then provide them a suitable amount of food at the end of each day to stabilize their weight at $85 \%$ of their freefeeding weight. We advise starting with $2 \mathrm{~g} /$ mouse/day and adapting the amount of food depending on the weight change of each mouse. Add some food pellets in their home cage to get them used to eating it.

B. Habituation period

It is important to note that this habituation can be done in other conditions because this first step consists of getting the mice used to being manipulated, eating food pellets, and stabilizing their weights. Therefore, using paradigms other than operant chambers is also possible (e.g., a two arms maze or Barnes maze with food at the end of each arm or in each hole). As an example, our lab uses operant chambers. In this section, we describe the procedure that we used.

A.

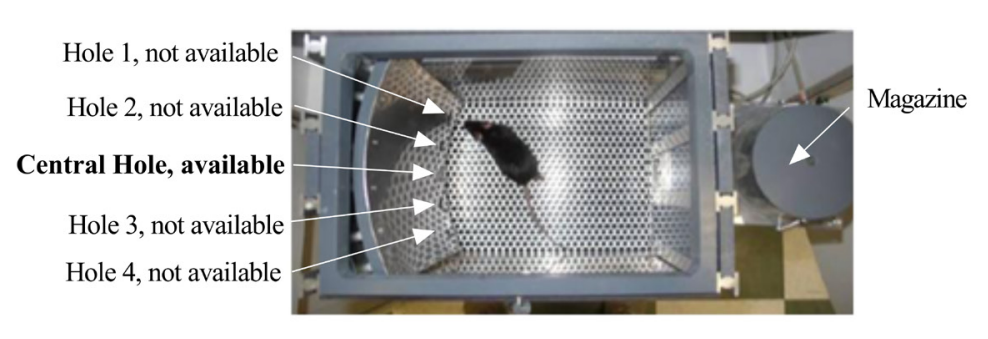

B.

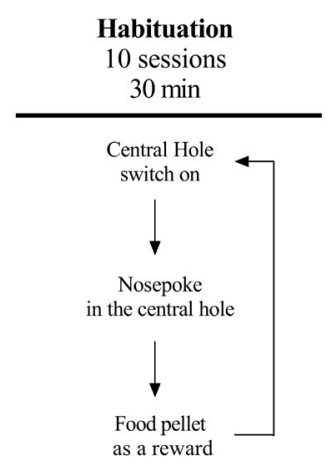

Figure 2. Apparatus and proceedings of the habituation period. A. Photograph of one operant chamber. B. Sequence of events during habituation in the operant chamber. When a mouse pokes its nose in the central hole, it triggers the food pellet dispenser and the central hole is turned off. As soon as the animal visits the magazine, the central hole is switched on again and the animal can do another nose poke.

Experimental procedure: Each time the mouse pokes in the central hole, a food pellet is delivered in the magazine (Figure 2B). Mice undergo one $30 \mathrm{~min}$ habituation session a day. Between each mouse the operant chamber is cleaned with a solution of $10 \%$ alcohol. In 10 days, we usually observe an increase in the amount of pellets eaten (from $\sim 5$ to $\sim 60$ food pellets), which shows that animals are learning the task and are getting used to eating in the experimental paradigm. As the amount of pellets earned increases across days, the amount of food given in their home cage decreases to maintain food motivation and weight.

C. The mouse gambling task

Since the experiment can take 10 to 30 min per mouse, and two sessions are done for each mouse per day, we advise doing the task with a maximum of 9 mice per week once the procedure is 
mastered.

1. Prepare quinine pellets: One week before starting the Mouse Gambling Task, prepare the quinine pellets. To do so, put around 20 dustless food pellets in a plastic container (e.g., Weighing Dishes). Add a $180 \mathrm{mM}$ solution of quinine with a plastic Pasteur pipette $(0.2 \mathrm{ml})$ until the liquid covers all of the pellets (Van Den Bos et al., 2006). Mix the liquid with the pellets until they all become darker (wet). Next, quickly remove any excess liquid and separate the pellets from one another to avoid sticking while drying. Let them dry overnight. For one week (maximum 9 mice), prepare around 50 quinine pellets.

Note: Usually, mice don't eat pellets previously soaked in the quinine solution because it is too bitter. If a mouse eats quinine pellets, you might have to exclude it from the group as it might not consider quinine as a penalty.

2. The task takes place in a maze with four transparent arms $(20 \mathrm{~cm}$ long $\times 10 \mathrm{~cm}$ wide) containing an opaque start box $(20 \mathrm{~cm} \times 20 \mathrm{~cm})$ and a choice area (Figure 3A). Use standard food pellets as a reward (dustless Precision Pellets, Grain-based, $20 \mathrm{mg}$, BioServ, New Jersey) and food pellets previously steeped in a $180-\mathrm{mM}$ solution of quinine as a penalty. The quinine pellets are unpalatable but not inedible. There are four different arms: two represent the long term "advantageous" choices and the other two the long term "disadvantageous" choices. For each arm, an immediate reward is located at the end of the arm just in front of a bottle cap containing the delayed reward. In "advantageous" arms, mice systematically find 1 pellet ("small reward") placed in front of a bottle cap containing food pellets in 18 trials of the 20 trials and quinine pellets for the two remaining trials (Figures $3 \mathrm{~A}$ and $3 \mathrm{~B}$ ). In the "disadvantageous" arms, mice find two food pellets ("large reward") placed in front of a bottle cap containing quinine pellets in 19 trials of the 20 and food pellets for the remaining trial (Figures $3 \mathrm{~A}$ and $3 \mathrm{~B}$ ). Advantageous choices are at first less attractive than disadvantageous ones because of the small immediate reward ( 1 pellet vs. 2 pellets). Despite this apparent lower attractiveness, advantageous choices are advantageous in the long term because food pellets have a higher probability of being found than quinine pellets (18 trials out of 20). Conversely, disadvantageous choices are less advantageous in the long term because animals have a higher probability of finding quinine pellets than food pellets (19 trials out of 20). Therefore, mice have to favour the small immediate reward (advantageous choices) to obtain the highest amount of pellets possible at the end of the day. Each animal completes 20 trials a day: 10 trials (= 1 session) in the morning (between 09:00 AM and 01:00 PM) and 10 trials (=1 session) during the afternoon (between 02:00 PM and 06:30 PM, Pittaras et al., 2013, 2016a, 2016b and 2018). Clean the maze with a 10\% ethanol solution between each mouse. 
A.

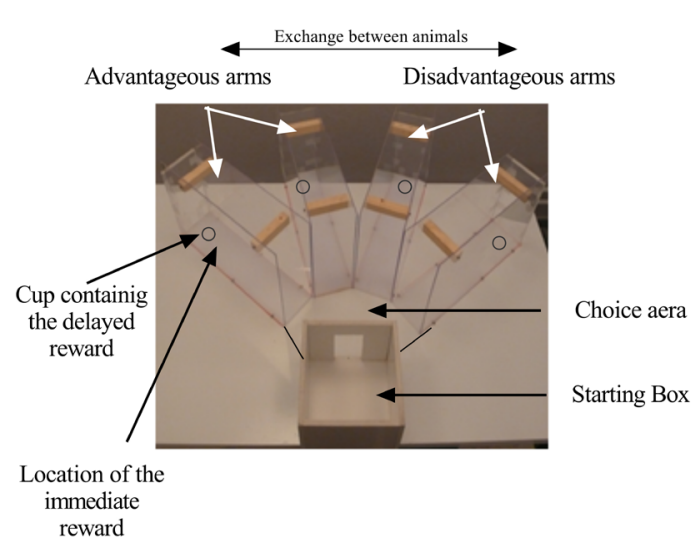

B.

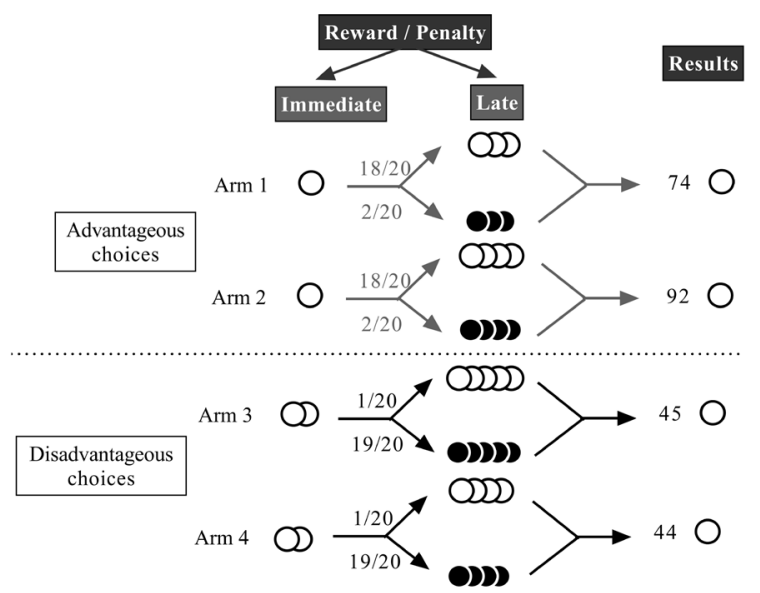

Figure 3. Apparatus and characteristics of the options of the Mouse Gambling Task. A. Photograph of the maze composed of four arms (two disadvantageous and two advantageous), a choice area and a starting box. B. Schematic representation of the MGT experimental design. White circles represent food pellets and black circles quinine pellets. Advantageous choices give access to one food pellet and disadvantageous choices give access to two food pellets. These immediate rewards are located at the end of the arm just in front of a bottle cap containing the delayed reward. Then the mouse can find three or four food pellets $(18 / 20)$ or quinine pellets $(2 / 20)$ in the advantageous choices, and can find four or five food pellets $(1 / 20)$ or quinine pellets $(19 / 20)$ in the disadvantageous choices. We distinguish advantageous choices from disadvantageous ones because mice earn more food pellets after 20 trials by choosing the advantageous ones. If a mouse only chooses arm 1 during the 20 trials, it will obtain 74 pellets ( $1 \times 20$ immediate food pellets reward and $18 \times 3$ food pellets as late reward). Likewise, if a mouse only chooses arms 2,3 , or 4 it will obtain 92,45 , or 44 food pellets respectively.

\section{Sequence of events of the Mouse Gambling Task}

a. Place the mouse in individual cage in the experimental room for a few minutes.

b. During the first morning session of the first day, randomly distribute 10 food pellets in the maze, and then place the mouse in the maze for $2 \mathrm{~min}$. If the mouse doesn't eat half of the available pellets, repeat this procedure during the afternoon of the first day. If the mouse eats more than half of the food pellets available, and for all the next morning and afternoon sessions of the task, put the mouse in the maze for 2 min before starting the task with no food pellet in it.

c. Place the mouse in the starting box inside an opaque cylinder during $15 \mathrm{~s}$ to avoid imposing direction to the mouse.

d. Take out the cylinder and start the timer.

e. Stop the timer when the mouse enters more than $3 / 4$ of its body into one arm. If it takes more than $2 \mathrm{~min}$, return to Step c. If it takes more than 2 min a second time, go directly to Step $\mathrm{g}$.

f. Score which arm is visited, the latency to make the choice, the number of food pellets eaten 
Please cite this article as: Pittaras et. al., (2020). The Mouse Gambling Task: Assessing Individual Decision-making Strategies in Mice,Bio-protocol 10

and the number of food pellets available to eat like the example in Figure 4.

g. Put the mouse back in the cylinder.

h. Clean the maze with distilled water to mix odors.

i. Prepare each arm for the next trial following Figure 4.

j. Go back to Step c until the mouse completes a total of 10 trials in the morning and 10 trials in the afternoon.

\section{Notes:}

i. As the amount of pellets eaten increases across days, the amount of food given in their home cage decreases to maintain food motivation and weight.

ii. Each day the quinine pellets for the advantageous arms and the food pellets for the disadvantageous arms need to be changed following a pseudo-random sequence (e.g., Figure 4). For one mouse, the position of the advantageous and disadvantageous arms remains the same during the 5 experimental days. Between mice, the position of the advantageous and disadvantageous arms is random (Figure $3 A$ ). The running order is changed every day. In other words, a mouse that starts the experimentation on Day 1 (9:00 AM) will be the second one on Day 2 (9:30 AM) to avoid a time-dependent behavior.

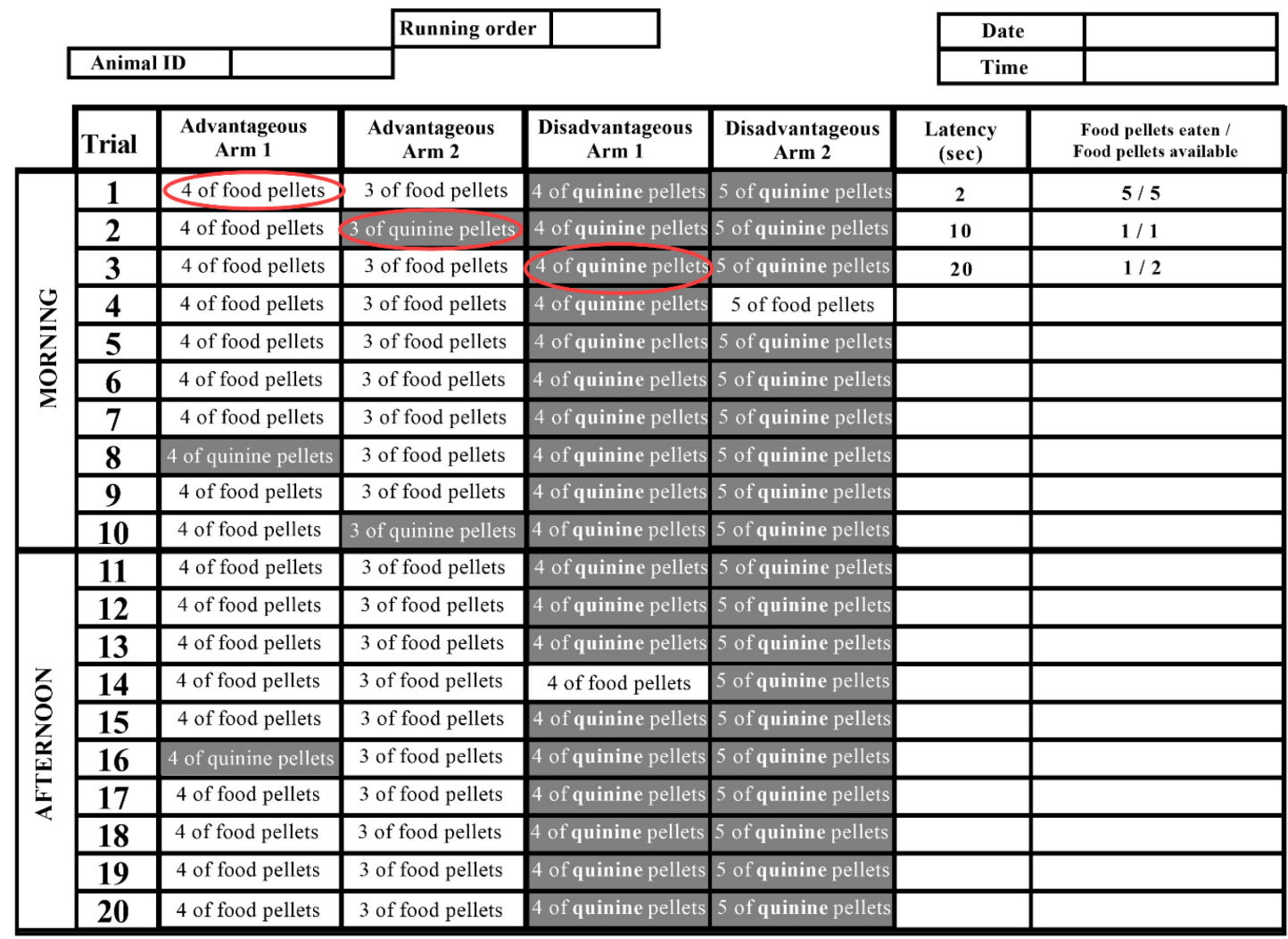

Figure 4. Picture example of table to fill during the task. This table is to fill for one day for one mouse that will do 10 trials in the morning and 10 trials in the afternoon. The first three trials are filled as an example. During the first trial, the mouse chooses the advantageous arm 1 in 2 
$s$ and eats 5 food pellets from the 5 food pellets available: 1 food pellet in front of the bottle cap (immediate reward) and 4 food pellets inside the bottle cap (late reward).

\section{Data analysis}

1. Global analysis

We score the percentage of advantageous choices by day [(number of advantageous choices/number of total choices) $x$ 100], the food pellet consumption (pellets earned), the number of quinine pellets obtained (but not eaten) and the latency to choose the arm during trials. The percentage of advantageous choices with control mice always increases across sessions from chance level to around $70 \%$ of advantageous choices (Figure $5 \mathrm{~A}$ ). We also calculate a rigidity score by measuring how many times the mouse chooses the same arm. For example, a rigidity score of $25 \%$ means that the mouse chooses the arm by chance and a rigidity score of $100 \%$ means that the mouse always chooses the same arm. Therefore, a rigidity score of $50 \%$ reflects that the mouse chooses one arm twice as much as the others, and a rigidity score of $75 \%$ that animal chooses one arm three times more often than the others (Pittaras et al., 2013, 2016a, 2016b, 2018).

2. Inter-individual decision-making strategies analysis

Another interesting characteristic of this protocol is that it allows the study of inter-individual variability during decision-making processes in mice (Pittaras et al., 2016a, 2016b and 2018). To do so, we first calculate the mean advantageous choices of the last 30 trials for each animal. Then, we use the K-mean clustering analysis (with Statistica software, version 12, Timmerman et al., 2013). This method distributes each animal to a set of animals that had the closest mean to its own preference value at the end of the task. It is possible to choose the number of groups but we advise separating animals into three typically-observed subgroups (Pittaras et al., 2013, 2016a, 2016b and 2018): animals that choose a majority of advantageous options at the end of the experiments, called "safe"; animals that explore options until the end of the experiment, called "risky"; and animals that maintain some exploration of available options but favor advantageous options, called "average" (Figure 5B). Interestingly, we have shown previously that these three subgroups are always present in a large group of mice $(n=54)$ following a Gaussian distribution (with safe and risky as extreme groups). Also, we have shown that these extreme subgroups might be more vulnerable to sleep debt (Pittaras et al., 2018) and could be more vulnerable to psychiatric disorders as they showed some specific traits of these diseases (Pittaras et al., 2016a and 2016b). 
A.

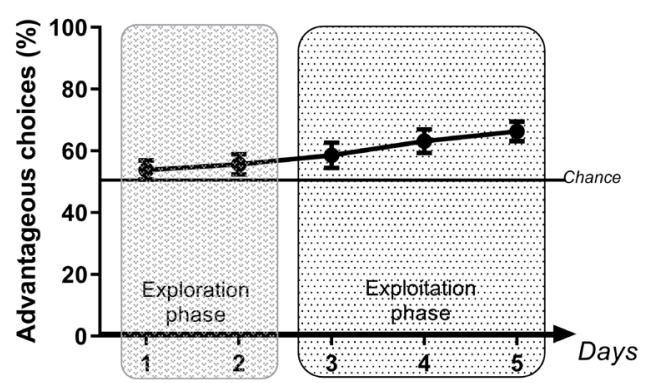

B.

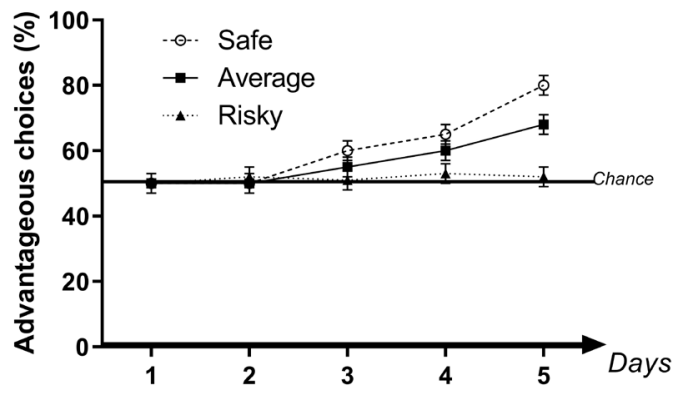

Figure 5. Example of the results usually obtained while doing the Mouse Gambling Task.

A. Schematic representation of the mean of the percentage of advantageous choices usually observed for control mice during 5 days of the MGT with an illustration of the exploration phase, during which mice explore options, and of an exploitation phase during which mice exploit their knowledge of the value of each option. During the exploration phase, mouse preferences are close to chance level while during the exploitation phase mice progressively prefer advantageous options. B. Schematic representation of the mean of the percentage of advantageous choices usually observed for the three control subgroups of mice.

\section{Notes}

1. Getting mice used to being manipulated by the person who will conduct the Mouse Gambling Task is important. It reduces stress, freezing and impulsive behavior during the Mouse Gambling Task which could hide the real decision-making strategy and hence lead to inappropriate results.

2. Food restricted animals housed by 3 or 4 could lead to some difficulties because of potential home cage dominancy. Give food to a mouse alone in another cage for $1 \mathrm{~h}$ if you observe that this mouse is losing more weight than others in the same cage.

3. Bringing mice close to the experimental room $30 \mathrm{~min}$ before starting reduces stress during the task.

4. If you observe that a mouse is eating the quinine pellets several times:

a. Change the quinine pellets, the quinine might not taste as strong anymore,

b. Be sure that the mouse's weight is not too low,

c. If the mouse continues to eat quinine pellets (unusual), you might have to exclude it from the group as it does not consider quinine as a penalty.

5. Technical tips:

a. Preparing the eight possible options in 8 different bottle caps (one bottle cap with 3 food pellets, one bottle cap with 3 quinine pellets, two bottle cap with 4 food pellets and two bottle caps with 4 quinine pellets, one bottle caps with 5 food pellets and one bottle cap with 5 quinine pellets) before starting helps to change quickly between two trials instead of changing the pellets inside the bottle caps for each trial. 
Please cite this article as: Pittaras et. al., (2020). The Mouse Gambling Task: Assessing Individual Decision-making Strategies in Mice,Bio-protocol 10

b. Don't always use the same bottle caps for the quinine and food pellets to ensure that there is a mixture of odors in each bottle cap.

\section{Acknowledgments}

We thank all the people who help setting up this behavioral task: Arnaud Cressant, Pierre Serreau, Jessica Bruijel, Françoise Dellu-Hagedorn, Betty Poly. We also thank Nathan Fisher for his help during the writing process.

\section{Competing interests}

Authors have no conflicts of interest or competing interests to declare.

\section{Ethics}

Animals are treated according to the ethical standards defined by the "Centre National de la Recherche Scientifique" for animal health and care with strict compliance with the EEC recommendations (no. 86/609).

\section{References}

1. Bechara, A., Damasio, A. R., Damasio, H. and Anderson, S. W. (1994). Insensitivity to future consequences following damage to human prefrontal cortex. Cognition 50(1-3): 7-15.

2. de Laat, B., Weerasekera, A., Leurquin-Sterk, G., Bormans, G., Himmelreich, U., Casteels, C. and Van Laere, K. (2018). Glutamatergic biomarkers for cocaine addiction: A longitudinal study using MR spectroscopy and mGluR5 PET in self-administering rats. J Nucl Med 59(6): 952-959.

3. de Visser, L., Homberg, J. R., Mitsogiannis, M., Zeeb, F. D., Rivalan, M., Fitoussi, A., Galhardo, V., van den Bos, R., Winstanley, C. A. and Dellu-Hagedorn, F. (2011a). Rodent versions of the iowa gambling task: opportunities and challenges for the understanding of decision-making. Front Neurosci 5: 109.

4. de Visser, L., Baars, A. M., van 't Klooster, J. and van den Bos, R. (2011b). Transient inactivation of the medial prefrontal cortex affects both anxiety and decision-making in male wistar rats. Front Neurosci 5: 102.

5. de Visser, L., Baars, A., Lavrijsen, M., VanderWeerd, C. and VandenBos, R. (2011c). Decision making performance is related to levels of anxiety and differential recruitment of frontostriatal areas in male rats. Neuroscience 184: 97-106.

6. Ferland, J. N. and Winstanley, C. A. (2017). Risk-preferring rats make worse decisions and show increased incubation of craving after cocaine self-administration. Addict Biol 22(4): 991-1001. 
Please cite this article as: Pittaras et. al., (2020). The Mouse Gambling Task: Assessing Individual Decision-making Strategies in Mice,Bio-protocol 10

7. Ferland, J. N., Carr, M. R., Lee, A. M., Hoogeland, M. E., Winstanley, C. A. and Pattij, T. (2018). Examination of the effects of cannabinoid ligands on decision making in a rat gambling task. Pharmacol Biochem Behav 170: 87-97.

8. Fitoussi, A., Le Moine, C., De Deurwaerdere, P., Laqui, M., Rivalan, M., Cador, M. and DelluHagedorn, F. (2015). Prefronto-subcortical imbalance characterizes poor decision-making: neurochemical and neural functional evidences in rats. Brain Struct Funct 220(6): 3485-3496.

9. Georgiou, P., Zanos, P., Bhat, S., Tracy, J. K., Merchenthaler, I. J., McCarthy, M. M. and Gould, T. D. (2018). Dopamine and stress system modulation of sex differences in decision making. Neuropsychopharmacology 43(2): 313-324.

10. Gueye, A. B., Trigo, J. M., Vemuri, K. V., Makriyannis, A. and Le Foll, B. (2016). Effects of various cannabinoid ligands on choice behaviour in a rat model of gambling. Behav Pharmacol 27(2-3 Spec Issue): 258-69.

11. Homberg, J. R., van den Bos, R., den Heijer, E., Suer, R. and Cuppen, E. (2008). Serotonin transporter dosage modulates long-term decision-making in rat and human. Neuropharmacology 55(1): 80-84.

12. Koot, S., Zoratto, F., Cassano, T., Colangeli, R., Laviola, G., van den Bos, R. and Adriani, W. (2012). Compromised decision-making and increased gambling proneness following dietary serotonin depletion in rats. Neuropharmacology 62(4): 1640-1650.

13. Koot, S., Baars, A., Hesseling, P., van den Bos, R. and Joels, M. (2013). Time-dependent effects of corticosterone on reward-based decision-making in a rodent model of the lowa Gambling Task. Neuropharmacology 70: 306-315.

14. Loi, M., Mossink, J. C., Meerhoff, G. F., Den Blaauwen, J. L., Lucassen, P. J. and Joels, M. (2017a). Effects of early-life stress on cognitive function and hippocampal structure in female rodents. Neuroscience 342: 101-119.

15. Loi, M., Sarabdjitsingh, R. A., Tsouli, A., Trinh, S., Arp, M., Krugers, H. J., Karst, H., van den Bos, R. and Joels, M. (2017b). Transient prepubertal mifepristone treatment normalizes deficits in contextual memory and neuronal activity of adult male rats exposed to maternal deprivation. eNeuro 4(5). doi: 10.1523/ENEURO.0253-17.2017.

16. Milienne-Petiot, M., Groenink, L., Minassian, A., Young, J. W. (2017). Blockade of dopamine D1family receptors attenuates the mania-like hyperactive, risk-preferring, and high motivation behavioral profile of mice with low dopamine transporter levels. J Psychopharmacol. 31(10):1334-1346.

17. Pais-Vieira, M., Mendes-Pinto, M. M., Lima, D. and Galhardo, V. (2009). Cognitive impairment of prefrontal-dependent decision-making in rats after the onset of chronic pain. Neuroscience 161(3): 671-679.

18. Peña-Oliver, Y., Sanchez-Roige, S., Stephens, D. N. and Ripley, T. L. (2014). Alpha-synuclein deletion decreases motor impulsivity but does not affect risky decision making in a mouse Gambling Task. Psychopharmacology (Berl) 231(12): 2493-2506. 
Please cite this article as: Pittaras et. al., (2020). The Mouse Gambling Task: Assessing Individual Decision-making Strategies in Mice,Bio-protocol 10

19. Pittaras, E., Cressant, A., Serreau, P., Bruijel, J., Dellu-Hagedorn, F., Callebert, J., Rabat, A. and Granon, S. (2013). Mice gamble for food: individual differences in risky choices and prefrontal cortex serotonin. J Addict Res Ther S4: 011.

20. Pittaras, E., Callebert, J., Chennaoui, M., Rabat, A. and Granon, S. (2016a). Individual behavioral and neurochemical markers of unadapted decision-making processes in healthy inbred mice. Brain Struct Funct 221(9): 4615-4629.

21. Pittaras, E., Faure, A., Leray, X., Moraitopoulou, E., Cressant, A., Rabat, A. A., Meunier, C., Fossier, P. and Granon, S. (2016b). Neuronal nicotinic receptors are crucial for tuning of E/l balance in prelimbic cortex and for decision-making processes. Front Psychiatry 7: 171.

22. Pittaras, E., Callebert, J., Dorey, R., Chennaoui, M., Granon, S. and Rabat, A. (2018). Mouse Gambling Task reveals differential effects of acute sleep debt on decision-making and associated neurochemical changes. Sleep 41(11). doi: 10.1093/sleep/zsy168.

23. Rivalan, M., Ahmed, S. H. and Dellu-Hagedorn, F. (2009). Risk-prone individuals prefer the wrong options on a rat version of the lowa Gambling Task. Biol Psychiatry 66(8): 743-749.

24. Rivalan, M., Valton, V., Series, P., Marchand, A. R. and Dellu-Hagedorn, F. (2013). Elucidating poor decision-making in a rat gambling task. PLoS One 8(12): e82052.

25. Sanchez-Roige, S., Ripley, T. L. and Stephens, D. N. (2015). Alleviating waiting impulsivity and perseverative responding by mu-opioid receptor antagonism in two inbred mouse strains. Psychopharmacology (Berl) 232(8): 1483-1492.

26. Timmerman, M. E., Ceulemans, E., De Roover, K. and Van Leeuwen, K. (2013). Subspace Kmeans clustering. Behav Res Methods 45(4): 1011-1023.

27. van den Bos, R., Lasthuis, W., den Heijer, E., van der Harst, J. and Spruijt, B. (2006). Toward a rodent model of the lowa gambling task. Behav Res Methods 38(3): 470-478.

28. van den Bos, R., Jolles, J., van der Knaap, L., Baars, A. and de Visser, L. (2012). Male and female Wistar rats differ in decision-making performance in a rodent version of the lowa Gambling Task. Behav Brain Res 234(2):375-9.

29. van den Bos, R., Koot, S. and de Visser, L. (2014). A rodent version of the lowa Gambling Task: 7 years of progress. Front Psychol 5: 203.

30. van Enkhuizen, J., Henry, B. L., Minassian, A., Perry, W., Milienne-Petiot, M., Higa, K. K., Geyer, M. A. and Young, J. W. (2014). Reduced dopamine transporter functioning induces high-reward risk-preference consistent with bipolar disorder. Neuropsychopharmacology 39(13): 3112-3122.

31. van Hasselt, F. N., de Visser, L., Tieskens, J. M., Cornelisse, S., Baars, A. M., Lavrijsen, M., Krugers, H. J., van den Bos, R. and Joels, M. (2012). Individual variations in maternal care early in life correlate with later life decision-making and c-fos expression in prefrontal subregions of rats. PLoS One 7(5): e37820.

32. Young, J. W., van Enkhuizen, J., Winstanley, C. A. and Geyer, M. A. (2011). Increased risktaking behavior in dopamine transporter knockdown mice: further support for a mouse model of mania. J Psychopharmacol 25(7): 934-943. 
Please cite this article as: Pittaras et. al., (2020). The Mouse Gambling Task: Assessing Individual Decision-making Strategies in Mice,Bio-protocol 10

33. Zeeb, F. D. and Winstanley, C. A. (2011). Lesions of the basolateral amygdala and orbitofrontal cortex differentially affect acquisition and performance of a rodent gambling task. J Neurosci 31(6): 2197-2204.

34. Zeeb, F. D., Robbins, T. W. and Winstanley, C. A. (2009). Serotonergic and dopaminergic modulation of gambling behavior as assessed using a novel rat gambling task. Neuropsychopharmacology 34(10): 2329-2343. 